\title{
ALPORT SYNDROME
}

\section{ACEls delay RRT and increase lifespan in Alport syndrome}

Administration of angiotensinconverting-enzyme inhibitors (ACEIs) early in the course of Alport syndrome delays progression to renal failure and improves life expectancy, say researchers. In a study that followed patients with Alport syndrome for a mean of $>20$ years, Oliver Gross and colleagues from the European Alport Registry found that use of ACEIs delayed the median age at onset of renal replacement therapy (RRT) by 3 years in individuals with impaired renal function and by 18 years in individuals with proteinuria compared with their untreated relatives. "Our study reveals the first therapeutic option for patients with Alport syndrome; dialysis can be delayed by ACEIs in a time-dependent manner. The earlier ACEI therapy starts, the better", explains Gross.
Alport syndrome is a hereditary disease that is characterized by progressive renal failure and leads to end-stage renal disease. The disease can be diagnosed early in childhood, but therapies to slow the progression of renal failure are lacking. Previous findings demonstrating the ability of ACEIs to delay renal failure in a mouse model of Alport syndrome led Gross and colleagues to establish the European Alport Registry, supported by the Association pour l'Information et la Recherche sur les Maladies Rénales Génétiques, to collate data on several generations of families affected by Alport syndrome and to investigate the effects of ACEIs on progression of renal failure and life expectancy in these individuals.

The researchers categorized 283 patients according to their renal function

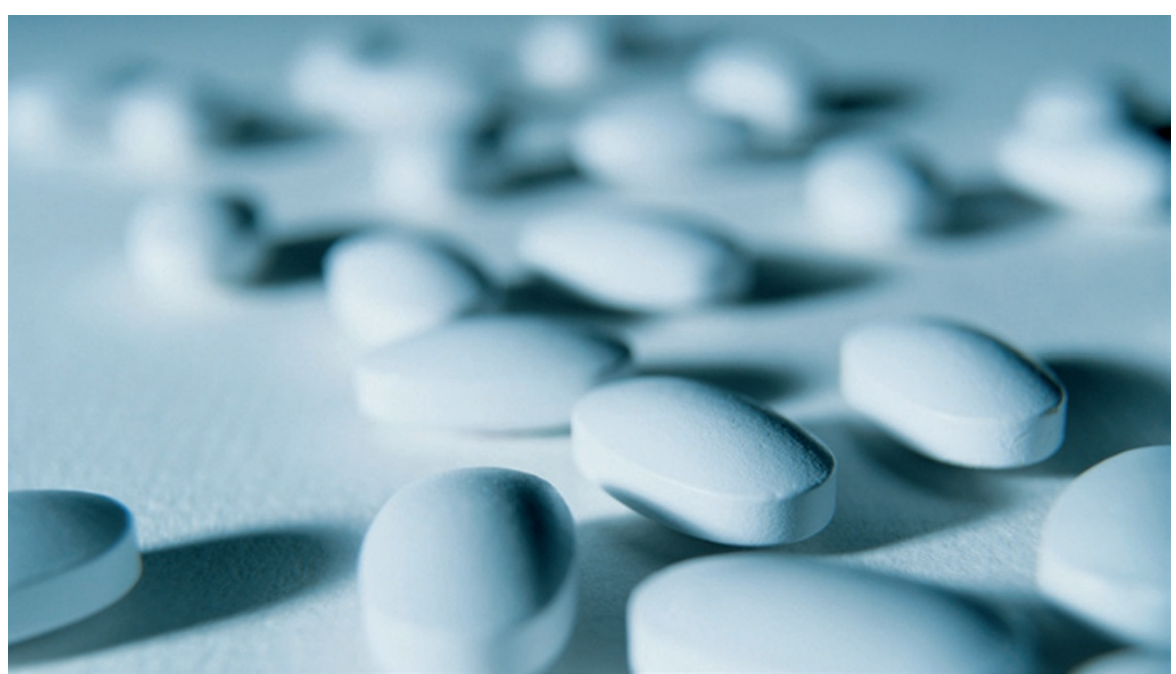

at the initiation of therapy-hematuria or microalbuminuria $(n=33)$, proteinuria $(n=115)$, impaired renal function $(n=26)$ and untreated relatives of treated patients $(n=109)$. They found that patients who did not receive ACEIs started RRT at a median age of 22 years. Patients with impaired renal function started on ACEIs at a median age of 20 years and started RRT at a median age of 25 years, whereas patients with proteinuria started on ACEIs at a median age of 13 years and started RRT at a median age of 40 years. Patients with hematuria or microalbuminuria started on ACEIs at a median age of 8 years. According to the researchers, none of these patients have yet required RRT. Gross et al. also found that ACEIs increased life expectancy beyond the median age of 55 years associated with no treatment. "Our data support the general need for early diagnosis and preventive therapy in all chronic kidney diseases in as yet oligosymptomatic patients", states Gross.

The researchers say that future prospective trials must focus on the risk-benefit balance of early therapy in oligosymptomatic children with Alport syndrome and on identifying prognostic factors to evaluate disease progression.

Susan J. Allison

Original article Gross, 0. et al. Early angiotensin-converting enzyme inhibition in Alport syndrome delays renal failure and improves life expectancy. Kidney Int. doi:10.1038/ ki.2011.407 